\title{
Raman spectroscopy is sensitive to biochemical changes related to various cartilage injuries
}

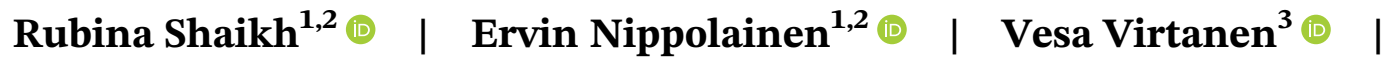

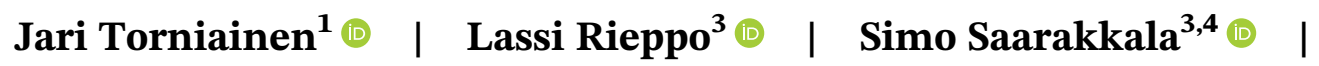 \\ Isaac O. Afara ${ }^{1,5}$ () | Juha Töyräs,
}

${ }^{1}$ Department of Applied Physics, University of Eastern Finland, Kuopio, Finland

${ }^{2}$ Department of Orthopedics

Traumatology and Hand Surgery, Kuopio

University Hospital, Kuopio, Finland

${ }^{3}$ Research Unit of Medical Imaging, Physics and Technology, University of Oulu, Oulu, Finland

${ }^{4}$ Department of Diagnostic Radiology, Oulu University Hospital, Oulu, Finland

${ }^{5}$ School of Information Technology and Electrical Engineering, The University of Queensland, Brisbane, Australia

${ }^{6}$ Diagnostic Imaging Centre, Kuopio

University Hospital, Kuopio, Finland

\section{Correspondence}

Rubina Shaikh, Ph.D., Department of Applied Physics, University of Eastern Finland, Finland, P.O. Box 1627, 70211.

Kuopio, Finland.

Email: rubinas@uef.fi

\section{Funding information}

Academy of Finland, Grant/Award Number: 315820; Doctoral Programme in Science, Technology, and Computing (SCITECO) of University of Eastern Finland; Kuopio University Hospital, Grant/Award Number: 5203111; The MIRACLE project-Horizon 2020 research and innovation programme-H2020-ICT2017-1, Grant/Award Number: 780598

\begin{abstract}
Raman spectroscopy is promising in vivo tool in various biomedical applications; moreover, in recent years, its use for characterizing articular cartilage degeneration has been developing. It has also shown potential for scoring the severity of cartilage lesions, which could be useful in determining the optimal treatment strategy during cartilage repair surgery. However, the effect of different cartilage injury types on Raman spectra is unknown. This study aims to investigate the potential of Raman spectroscopy for detecting changes in cartilage due to different injury types. Artificial injuries were induced in cartilage samples using established mechanical and enzymatic approaches to mimic trauma-induced and natural degeneration. Mechanical damage was induced using surface abrasion (ABR, $n=12$ ) or impact loading (IMP, $n=12$ ), while enzymatic damage was induced using three different treatments: $30 \mathrm{~min}$ trypsin digestion (T30, $n=12$ ), 90 min collagenase digestion (C90, $n=12$ ), and $24 \mathrm{~h}$ collagenase digestion $(\mathrm{C} 24, n=12)$. Raman spectra were obtained from all specimens, and partial least squares discriminant analysis (PLS-DA) was used to distinguish cartilage injury types from their respective controls. PLS-DA cross-validation accuracies were higher for C24 (88\%) and IMP (79\%) than for C90 (67\%), T30 (63\%), and ABR (58\%) groups. This study indicates that Raman spectroscopy, combined with multivariate analysis, can discern different cartilage injury types. This knowledge could be useful in clinical decision-making, for example, selecting the optimal treatment remedy during cartilage repair surgery.

\section{KE Y W O R D S}

cartilage degeneration, multivariate analysis, osteoarthritis, PLS-DA, Raman spectroscopy
\end{abstract}




\section{1 | INTRODUCTION}

Articular cartilage (AC) is a specialized connective tissue that allows near frictionless movement of joints. ${ }^{[1]} \mathrm{AC}$ is an avascular and aneural tissue consisting predominantly of water $(65 \%-80 \%)$, collagen $(10 \%-30 \%)$, and proteoglycans $(3 \%-10 \%){ }^{[1]}$ Due to the limited regenerative capacity of $\mathrm{AC}$, it is susceptible to progressive degeneration after an initial injury. ${ }^{[2]}$ Impact injury on the joint can lead to a degenerative joint condition known as posttraumatic osteoarthritis (PTOA), which in its advanced stage is characterized by erosion of cartilage matrix, joint pain, and restricted mobility. ${ }^{[3]}$ Hence, it is critical to diagnose joint damage at an early stage where matrix degeneration may be halted or even reversed using pharmaceutical or surgical interventions. ${ }^{[3-5]}$ Cartilage injury can be evaluated and repaired during arthroscopic surgery. However, conventional arthroscopy suffers from poor diagnostic reproducibility and lacks reliable quantitative and objective information. ${ }^{[6,7]}$ It has been suggested that the reliability and objectivity of arthroscopic evaluation can be improved by complementary quantitative diagnostic optical techniques-such as near-infrared spectroscopy, Fourier-transform infrared spectroscopy (FTIR), Raman spectroscopy, and optical coherence tomography. These methods can be adapted in a minimally invasive approach for nondestructive, sensitive, and objective assessment of cartilage integrity. ${ }^{[8-12]}$ Furthermore, Raman spectroscopy is also a potential technique for quantitative arthroscopic evaluation of joint tissues. ${ }^{[13-21]}$

Raman spectroscopy is based on inelastic scattering (i.e., Raman scattering) of light, enabling assessment of the biochemical composition and, thus, the integrity of biological tissues. ${ }^{[22]}$ Initially, after the discovery of Raman scattering, the major limiting factor for the implementation of Raman spectroscopy in biomedicine was the weak scattering signals. ${ }^{[23]}$ Nevertheless, the invention of the laser in the 1960s and application of fiber optics and charge-coupled devices (CCDs) in the 1980s has rapidly increased the popularity of Raman spectroscopy in biomedical research. ${ }^{[13,23,24]}$ In the last decades, Raman spectroscopy has proved to be a promising tool for characterizing biomolecular changes in tissue composition related to various diseases. ${ }^{[13,18,23-25]}$ In comparison with other vibrational spectroscopic techniques, Raman spectroscopy encounters less interference from water in the biological fingerprint region $\left(800^{-1}\right.$ $\left.1800 \mathrm{~cm}^{-1}\right){ }^{[16]}$ This asset makes Raman spectroscopy particularly suitable for the characterization of biomolecular changes in biological tissues, such as AC, which consists of $65 \%-80 \%$ water. $^{[1,16]}$
Raman spectroscopy has been shown to be a potential technique for quantifying cartilage properties. ${ }^{[18]}$ The study explored its potential for arthroscopic assessment of $\mathrm{AC}^{\left[{ }^{[1]}\right.}$ In the same year, Raman spectroscopy was shown to be capable of detecting biomolecular changes associated with impact-related cartilage damage. ${ }^{[16]}$ Subsequently, it was also suggested that Raman spectroscopy has the potential for diagnosing cartilage damage, as well as monitoring subchondral bone integrity in pathogenesis related to osteoarthritis (OA). ${ }^{[19]}$ Moreover, a Raman band that might be useful for the determination of proteoglycans in cartilage and bone was identified. ${ }^{[15]}$ Molecular information derived from Raman spectroscopy could be linked with the conventional Collins scale, providing a potential nondestructive approach for pathological grading of cartilage and subchondral bone changes in OA. ${ }^{[20]}$ Additionally, it has been demonstrated that Raman spectroscopy can detect depth-dependent changes in bovine cartilage after a wear test. ${ }^{[21]}$ Finally, it has been shown that Raman bands at high wavenumbers $\left(3,000-3,800 \mathrm{~cm}^{-1}\right)$ are associated with water content, which has a significant correlation with the mechanical properties of human cartilage. ${ }^{[26]}$

Based on the literature, Raman spectroscopy can identify biochemical changes in cartilage matrix due to mechanical damage $;^{[16,21]}$ however, changes in the Raman spectrum related to selective enzymatic degradation have not been thoroughly investigated. This information related to various kinds of cartilage injuries could be crucial when selecting the optimal treatment methods during arthroscopic cartilage repair surgery. However, the sensitivity of Raman spectroscopy to different types of cartilage pathology needs to be further investigated before exploring its full potential in the arthroscopic characterization of cartilage integrity. We hypothesize that Raman spectroscopy can detect biochemical changes associated with cartilage injuries induced mechanically or enzymatically, which mimic post-traumatic and idiopathic OA, respectively.

The validity of this hypothesis was investigated experimentally using samples with various types of mechanical and enzymatic degeneration. Mechanical damage was induced through surface abrasion (ABR) or impact loading (IMP), whereas enzymatic damage was caused using three different treatments-30 min trypsin digestion (T30), 90 min collagenase digestion (C90), and $24 \mathrm{~h}$ collagenase digestion (C24). Raman spectra were measured from the specimens, and partial least squares discriminant analysis (PLS-DA) was carried out to differentiate damaged samples from their controls, as well as to differentiate between injury types, based on spectral variations resulting from the different cartilage injuries (Figure 1). 


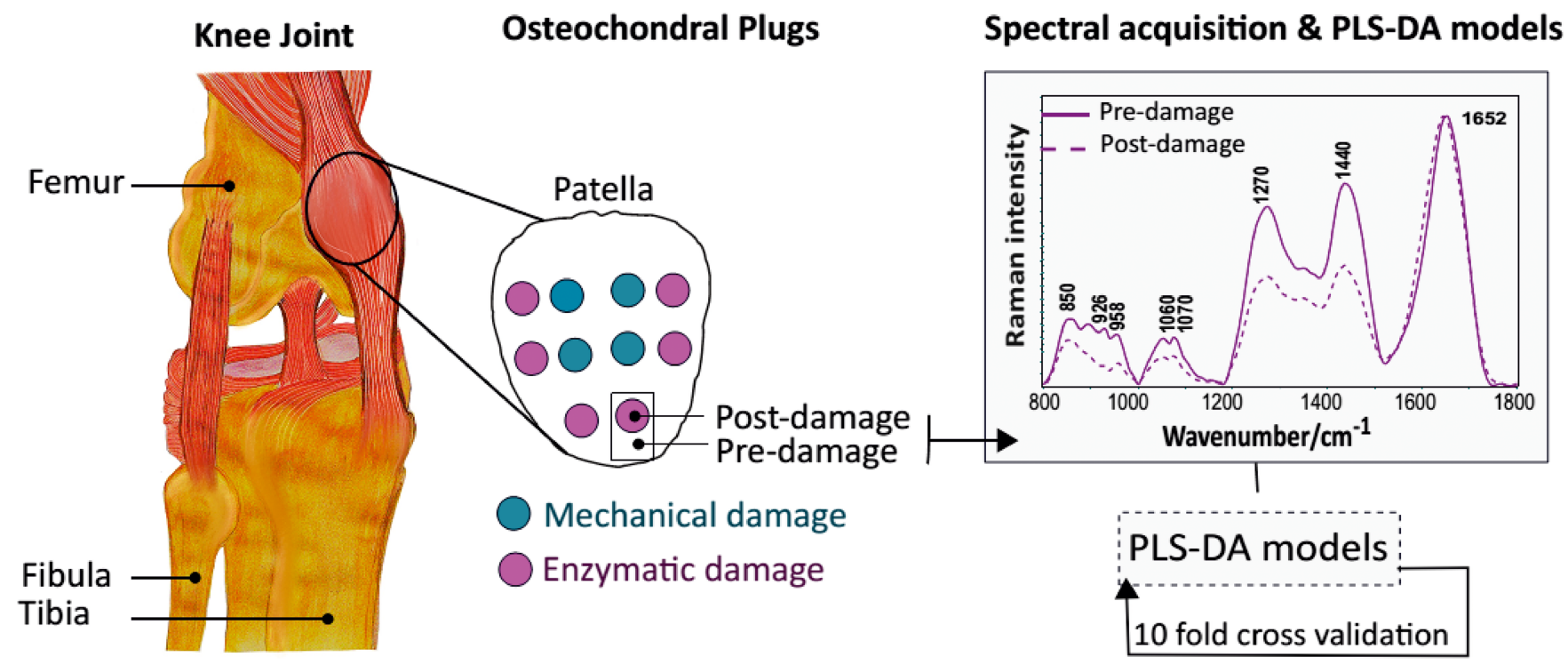

F I G U RE 1 Study protocol [Colour figure can be viewed at wileyonlinelibrary.com]

\section{2 | MATERIALS AND METHODS}

\subsection{Sample preparation}

Patellae $(n=10)$ were extracted from fresh bovine (age 14-22 months) knee joints obtained from a local slaughterhouse, and therefore, no ethical permission was required. Osteochondral specimens $(n=60)$ were extracted from different anatomical locations of the lateral and medial patellae without any signs of natural damage on the cartilage surface. One-half of each specimen was used as a pre-damage group, and the other half was utilized for post-damage (mechanically or enzymatically degraded cartilage) experiments (Figure 1). All the specimens (pre- and post-damage specimens) were stored in phosphate-buffered saline (PBS) between the preparation steps and the Raman spectra measurements step.

Pre- and post-damage specimens for each group were extracted from the same anatomical location to avoid variations based on the specimen's location on the patella. In the case of mechanical damage, cylindrical osteochondral specimens (diameter $=7 \mathrm{~mm}$ ) were prepared, whereas enzymatic degradation was carried out on larger tissue samples. Larger samples were utilized to avoid lateral penetration of enzyme into the sample. After the enzymatic treatment, cylindrical plugs (diameter $=7 \mathrm{~mm}$ ) were extracted, and Raman spectra were acquired from the region of interest. Specimens were always immersed in PBS before and after measurements. After spectral measurements, the specimens were formalin fixed, decalcified in ethylenediaminetetraacetic acid (EDTA), and then embedded in paraffin.
Histological sections (thickness $=3 \mu \mathrm{m}$ ) were cut and stained using Safranin-O, a stain that binds stoichiometrically with matrix proteoglycans. Digital images were acquired from the histological sections using a PathScanEnabler-IV (MeyerInstruments, Inc., USA).

Mechanical damage was induced on the cylindrical osteochondral specimens via impact loading (IMP, $n=12$ ) or surface abrasion (ABR, $n=12$ ). Surface abrasion and impact damage were created following the protocol described in earlier studies (Figure 2). ${ }^{9,11} \mathrm{~A}$ custom-made drop tower was used to create an impact injury on the specimens (Figure 2b). ${ }^{11}$ Impact loading was carried out using a stainless steel impactor (200 g) with a polished steel ball (diameter $=1 \mathrm{~cm}$ ) at the impact surface. The impactor was dropped from a height of $7.5 \mathrm{~cm}$. The impactor height, and thus, energy delivered to the cartilage surface, was determined based on preliminary assessments to create minor cracks on the cartilage surface. A customised setup (Figure 2a) was used to induce abrasive damage on the cartilage surface. ${ }^{9}$ Specimen surface was abraded under constant stress $(4 \mathrm{kPa})$ by a rotating metal plate $\left(180^{\circ}\right)$ covered with sandpaper (P80, $200 \mu \mathrm{m}$ particle size). This procedure was repeated in two perpendicular directions. Immediately after this protocol, specimens were rinsed in PBS, and subsequently, their Raman spectra were acquired.

\section{2 | Enzymatic degradation}

Enzymatic degradation of specimens $(n=36)$ was conducted using either collagenase or trypsin. Collagenase D (0.1 mg/ml, Sigma-Aldrich Inc., St. Louis, MO, USA) was 
(a)

(b)

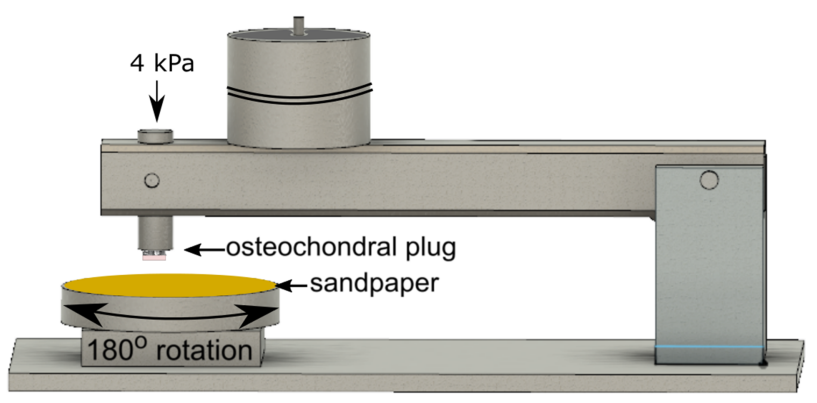

1

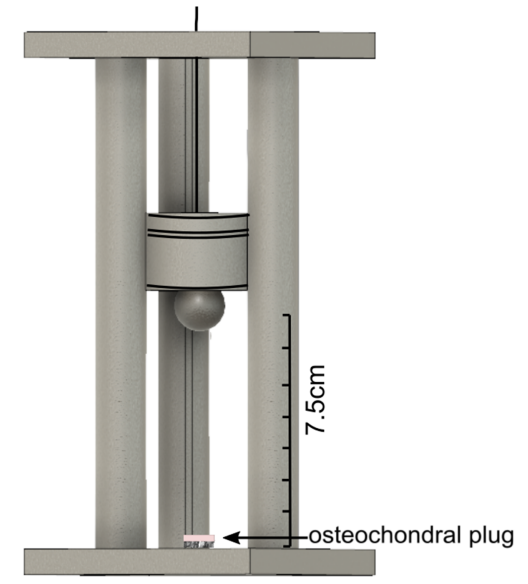

F I G URE 2 (a) Custom-made tool for inducing surface abrasion on cartilage. (b) Custom-made drop tower to create impact injury [Colour figure can be viewed at wileyonlinelibrary.com]

utilized for digestion of the collagen network, whereas trypsin $(0.5 \mathrm{mg} / \mathrm{ml}$, T4299, Sigma-Aldrich Inc., St. Louis, MO, USA) was used to deplete the proteoglycans from the extracellular matrix. ${ }^{27,28}$ Collagenases D explicitly targets and cleaves the collagen molecules in the triple helix region, whereas trypsin cuts the peptide bond on the C-terminal side of lysine and arginine amino acids (Sigma-Aldrich Inc).

Prior to enzymatic degradation, the specimens were subdivided into three groups: collagenase $24 \mathrm{~h}$ (C24, $n=12)$, collagenase $90 \mathrm{~min}(\mathrm{C} 90, n=12)$, and trypsin $30 \mathrm{~min}(\mathrm{~T} 30, n=12)$. Specimens were incubated at $37^{\circ} \mathrm{C}$ and $5 \% \mathrm{CO}_{2}$ in PBS solution containing the respective enzymes with supplementary antibiotics (PenicillinStreptomycin-Amphotericin B, 100 units/ml penicillin, $100 \mu \mathrm{g} / \mathrm{ml}$ streptomycin, and $0.25 \mu \mathrm{g} / \mathrm{ml}$ amphotericin B, Sigma-Aldrich Inc., St. Louis, MO, USA). ${ }^{29}$ For mild cartilage degradation, we applied a shorter incubation time of $30 \mathrm{~min}$ for trypsin (T30) and $90 \mathrm{~min}$ for collagenase (C90). A long incubation time of $24 \mathrm{~h}$ was also applied with collagenase (C24) to induce severe damage. After enzymatic treatment, specimens were rinsed in PBS before the Raman spectroscopic measurements.

\section{3 | Raman spectroscopy}

Raman spectra were acquired from the center of each osteochondral specimen three times. A total of 360 spectra from 60 damage (post-damaged) and 60 normal (predamaged) specimens were measured. Raman confocal microscope (Thermo Fisher Scientific DXR2xi, Madison, WI, USA) in the range of $200-3,400 \mathrm{~cm}^{-1}$ with $785 \mathrm{~nm}$ laser at $30 \mathrm{~mW}$ through a $10 \mathrm{x}$ objective, with a $50 \mu \mathrm{m}$ confocal pinhole, $0.5 \mathrm{~s}$ exposure time, and 120 accumulation was used.

\section{4 | Data pre-processing and multivariate analysis}

Multivariate data analysis was carried out on preprocessed spectra. Pre-processing of the acquired spectral data was consistent with a previously established protocol. ${ }^{30,31}$ The spectral pre-processing procedure was conducted using an open-source toolbox (https://github. com/uef-bbc/nippy). ${ }^{32}$ Pre-processing included the following: (1) background subtraction, (2) interpolation (800-1,800 $\mathrm{cm}^{-1}$ range), (3) baseline correction, and (4) SNV (standard normal variate) normalization.

PLS-DA was carried out to classify the cartilage samples into pre- versus post-damage groups. The investigation was based solely on the Raman spectral data and performed using a classification toolbox (version 5.3) in MATLAB (MathWorks Inc, Natick, MA, USA). ${ }^{33}$ PLS-DA models were developed to classify pre- versus postdamaged specimens of different injury types. Due to the relatively small sample size, 10 -fold cross-validation was used during development of the PLS-DA models. The performance of the models was evaluated based on crossvalidated error rates.

\section{3 | RESULTS}

The mean Raman spectra (background and baseline corrected) of all groups showed significant peaks at 1,652, 1,440 , and $1,270 \mathrm{~cm}^{-1}$ and minor peaks at 1,070, 1,060, 958, 926, and $850 \mathrm{~cm}^{-1}$ (Figures 3 and S2). These features correspond to the vibrational modes of collagen, glycosaminoglycan (GAGs), and bone (Table S1). We observed the most significant spectral differences between preversus post-damage in C24 and IMP groups (Figure 3). We also noticed subtle spectral differences at 1,652, 

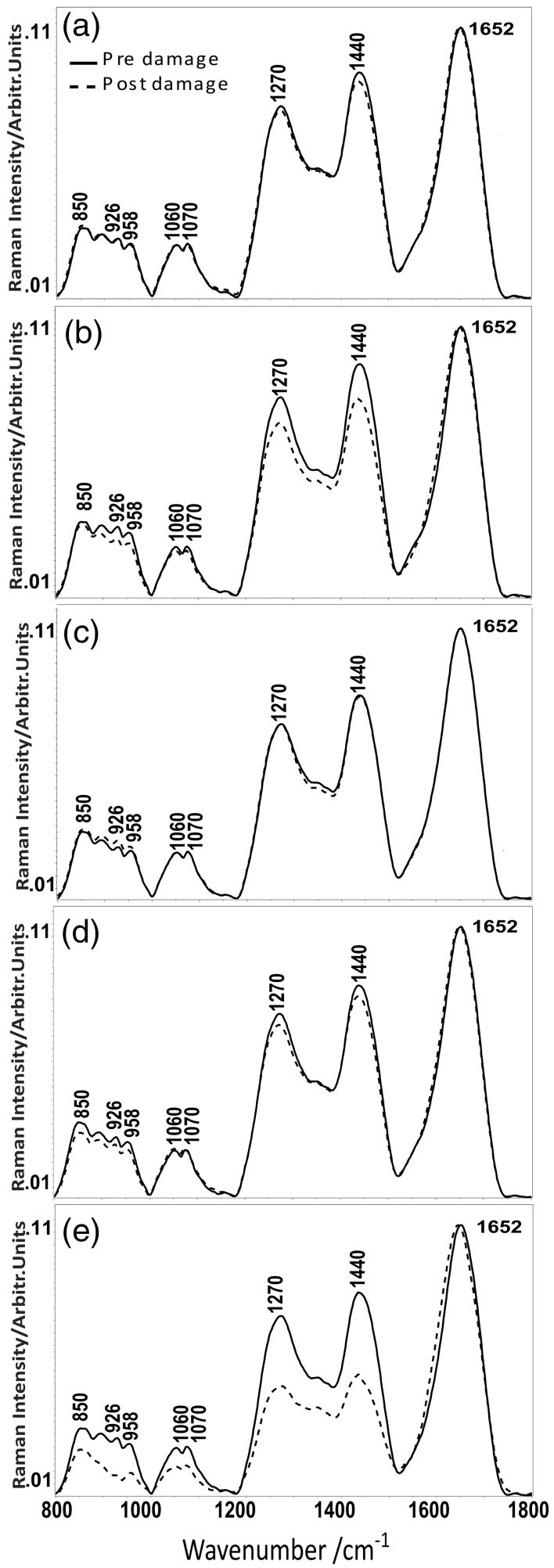

FIG URE 3 Mean Raman spectra of pre- versus post-cartilage damage (a) ABR (abrasion), (b) IMP (impact), (c) T30 (trypsin $30 \mathrm{~min}$ ), (d) C90 (collagenase $90 \mathrm{~min}$ ), and (e) C24 (collagenase $24 \mathrm{~h}$ )

$1,440,1,270$, and $800-1,100 \mathrm{~cm}^{-1}$ between pre-ABR versus post-ABR and $\mathrm{C} 90$ groups. Besides, we observed minor differences in the $850-1,000 \mathrm{~cm}^{-1}$ range due to trypsin degeneration (T30).
The PLS-DA calibration models had accuracies ranging between $81 \%$ and $100 \%$, with cross-validation accuracies ranging between $58 \%$ and $88 \%$ (Table 1). The best cross-validated accuracies were obtained between pre-C24 and post-C24 (88\%) and among pre-IMP and post-IMP (79\%) groups. PLS-DA classification accuracy for the "pre- versus post-cartilage damage" group was $74 \%$ (Figure 4a). In contrast, the classification models that discriminated mechanical and enzymatic damage from their respective controls showed classification accuracies of $81 \%$ and $76 \%$, respectively (Figure $4 \mathrm{~b}$ ).

Images of Safranin-O stained sections show that samples with $\mathrm{ABR}$ and impact damage have irregular cartilage surface and chondral cracks, respectively (Figure S1). We observed marginally reduced Safranin-O staining (i.e., mild proteoglycan loss) in the case of C90 and T30 groups. In the case of the $\mathrm{C} 24$ group, we noticed disorganization of cartilage in the superficial and middle zones with a significant reduction in safranin-O staining (i.e., severe proteoglycan loss).

\section{4 | DISCUSSION}

In the present study, we investigated the capability of Raman spectroscopy to detect changes in cartilage composition induced by mechanical or enzymatic degradation, mimicking post-traumatic and idiopathic OA, respectively. The mean Raman spectra of nondamaged cartilage revealed molecular vibrations corresponding to amide I, $\delta \mathrm{CH}_{2}$ stretch, amide III, carbonate stretch, $\mathrm{SO}_{3}{ }^{-}$ symmetric stretch, $\mathrm{PO}_{4}{ }^{3--}$ phosphate stretch, proline, and hydroxyproline (Table S1). These observations are in line with literature suggesting that molecular vibrations from collagen, GAGs, and bone dominate Raman spectra recorded from osteochondral specimens. ${ }^{14,17,19}$ The substantial differences in Raman spectral features between pre- and post-damage in C24 and IMP groups are due to the injury-related disruption in biochemical structure and composition. The mean Raman spectrum of the C24 group (Figure 3) exhibited a blue shift in the amide I peak from 1,652 to $1,642 \mathrm{~cm}^{-1}$; the change in the amide I can be attributed to secondary structural deformation of collagen. This shift in the amide I could not be assigned to proteoglycans as it was not observed in the T30 group. We notice a similar but more subtle change in the amide I peak of the $\mathrm{C} 90$ group. To the best of our knowledge, this has not been reported earlier.

Raman spectra displayed significant intensity-related variations in amide III and $\delta \mathrm{CH}_{2}$ stretch in an impact injury group. Earlier, the red-shift from 1,264 to $1,274 \mathrm{~cm}^{-1}$ after impact damage in porcine cartilage has been reported. ${ }^{16}$ It was proposed that this shift in 


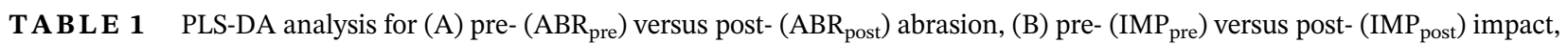
(C) pre- $\left(\mathrm{T} 30_{\text {pre }}\right)$ versus post- (T30 post $)$ trypsin $30 \mathrm{~min}$, (D) pre- $\left(\mathrm{C} 90_{\text {pre }}\right)$ versus post- $\left(\mathrm{C} 90_{\text {post }}\right)$ collagenase 90 min, (E) pre- (C24 pre versus post- $\left(\mathrm{C} 24_{\text {post }}\right)$ collagenase $24 \mathrm{~h}$, (F) pre- $\left(\mathrm{M}_{\text {pre }}\right)$ versus post- $\left(\mathrm{M}_{\text {post }}\right)$ mechanical damage, $(\mathrm{G})$ pre- $\left(\mathrm{E}_{\text {pre }}\right)$ versus post- $\left(\mathrm{E}_{\text {post }}\right)$ enzymatic damage, $(H)$ enzymatic damage $\left(E_{\text {post }}\right)$ versus mechanical damage $\left(M_{\text {post }}\right)$, and $(I)$ pre- $\left(D_{\text {pre }}\right)$ versus post- $\left(D_{\text {post }}\right)$ "damage" group

\begin{tabular}{|c|c|c|c|c|c|}
\hline \multicolumn{2}{|c|}{ Partial least squares discriminant analysis (PLS-DA) } & \multirow{2}{*}{$\begin{array}{l}\text { Number of LV } \\
4\end{array}$} & \multirow{2}{*}{$\begin{array}{l}\text { Sensitivity (\%) } \\
83\end{array}$} & \multirow{2}{*}{$\begin{array}{l}\text { Specificity (\%) } \\
92\end{array}$} & \multirow{2}{*}{$\begin{array}{l}\text { Accuracy (\%) } \\
91\end{array}$} \\
\hline (A) $\mathrm{ABR}_{\text {pre }}$ versus $\mathrm{ABR}_{\text {post }}$ & Calibration model & & & & \\
\hline & Cross-validation & & 67 & 50 & 58 \\
\hline \multirow{2}{*}{ (B) $\mathrm{IMP}_{\text {pre }}$ versus IMP $\mathrm{Iost}_{\text {pst }}$} & Calibration model & 4 & 100 & 100 & 100 \\
\hline & Cross-validation & & 83 & 75 & 79 \\
\hline \multirow{2}{*}{ (C) $\mathrm{T} 30_{\text {pre }}$ versus $\mathrm{T} 30_{\text {post }}$} & Calibration model & 4 & 83 & 92 & 88 \\
\hline & Cross-validation & & 58 & 67 & 63 \\
\hline \multirow[t]{2}{*}{ (D) $\mathrm{C} 90_{\text {pre }}$ versus $\mathrm{C} 90_{\text {post }}$} & Calibration model & 4 & 92 & 83 & 88 \\
\hline & Cross-validation & & 58 & 75 & 67 \\
\hline \multirow[t]{2}{*}{ (E) $\mathrm{C} 24_{p r e}$ versus $\mathrm{C} 24_{\text {post }}$} & Calibration model & 3 & 92 & 92 & 92 \\
\hline & Cross-validation & & 92 & 83 & 88 \\
\hline \multirow{2}{*}{ (F) $\mathrm{M}_{\text {pre }}$ versus $\mathrm{M}_{\text {post }}$} & Calibration model & 8 & 96 & 83 & 90 \\
\hline & Cross-validation & & 83 & 79 & 81 \\
\hline \multirow[t]{2}{*}{ (G) $\mathrm{E}_{\text {pre }}$ versus $\mathrm{E}_{\text {post }}$} & Calibration model & 8 & 83 & 92 & 88 \\
\hline & Cross-validation & & 81 & 72 & 76 \\
\hline \multirow[t]{2}{*}{ (H) $\mathrm{E}_{\text {post }}$ versus $\mathrm{M}_{\text {post }}$} & Calibration model & 8 & 92 & 92 & 92 \\
\hline & Cross-validation & & 75 & 79 & 77 \\
\hline \multirow[t]{2}{*}{ (I) $\mathrm{D}_{\text {pre }}$ versus $\mathrm{D}_{\text {post }}$} & Calibration model & 8 & 87 & 75 & 81 \\
\hline & Cross-validation & & 82 & 67 & 74 \\
\hline
\end{tabular}

Note: The bold number in the table is to emphasis the description in the results section. It will help the readers to have smooth access to the information.

amide III was due to compression of $\mathrm{C}-\mathrm{N}$ vibration in collagen fibers. ${ }^{16}$ In the current study, we noticed intensity-related variations in the amide III peak. These changes in amide III peak intensity are potentially due to the conformational, and configuration change in the collagen macromolecules as well as depletion of proteoglycans (Figure S1). We observed subtle but significant changes in amide III and $\delta \mathrm{CH} 2$ peaks in the Raman spectra of the ABR group. These observations are also consistent with an earlier study. ${ }^{21}$ The mean Raman spectrum of the T30 group exhibited minor variation in $\delta \mathrm{CH}_{2}$ stretch and amide III peak. We did not observe any differences at $1,060 \mathrm{~cm}^{-1}\left(\mathrm{SO}_{3}\right.$-symmetric stretch) - the molecular vibration assigned to chondroitin sulfate. These findings could be due to trypsin targeting the peptide bonds rather than the chondroitin chain. ${ }^{22}$

To investigate the statistical significance of Raman spectral variance induced by mechanical or enzymatic damage, PLS-DA was carried out. PLS-DA is a linear discriminant analysis that extends the properties of partial least squares regression to classification. In the current study, the observed classification accuracy was directly proportional to the severity of the cartilage damage (Table 1). Thus, the classification accuracy for pre- versus post-damage was highest for $\mathrm{C} 24$ and lowest for the ABR group. The low cross-validation accuracy of $58 \%$ observed in the case of ABR group is likely due to mild surface damage. It is widely thought that proteasesmediated degradation of cartilage matrix is a fundamental feature of idiopathic OA. ${ }^{34}$

In contrast, mechanical damage induces structural changes in cartilage matrix, leading to PTOA. ${ }^{3,35}$ Based on these facts, we combined the spectra from $A B R$ and IMP groups into mechanical damage group and T30, C90, and C24 groups into enzymatic damage group. We further pooled the spectra from mechanical and enzymatic degraded specimens into the "pre-damage $\left(D_{\text {pre }}\right)$ versus post-damage $\left(D_{\text {post }}\right)$ " group and observed that PLS-DA had a classification accuracy of $74 \%$ (Figure 4a). Moreover, we observed relatively similar classification accuracies for pre- versus post-damage for mechanical damage $(81 \%)$ and enzymatic damage $(76 \%)$ groups. The confusion matrix of 10-fold cross-validation for the PLS-DA analysis is presented in Table 2.

Safranin-O stain binds to matrix proteoglycans in cartilage tissue, and decrease in the intensity of the stain on histological slides is related to the loss of 


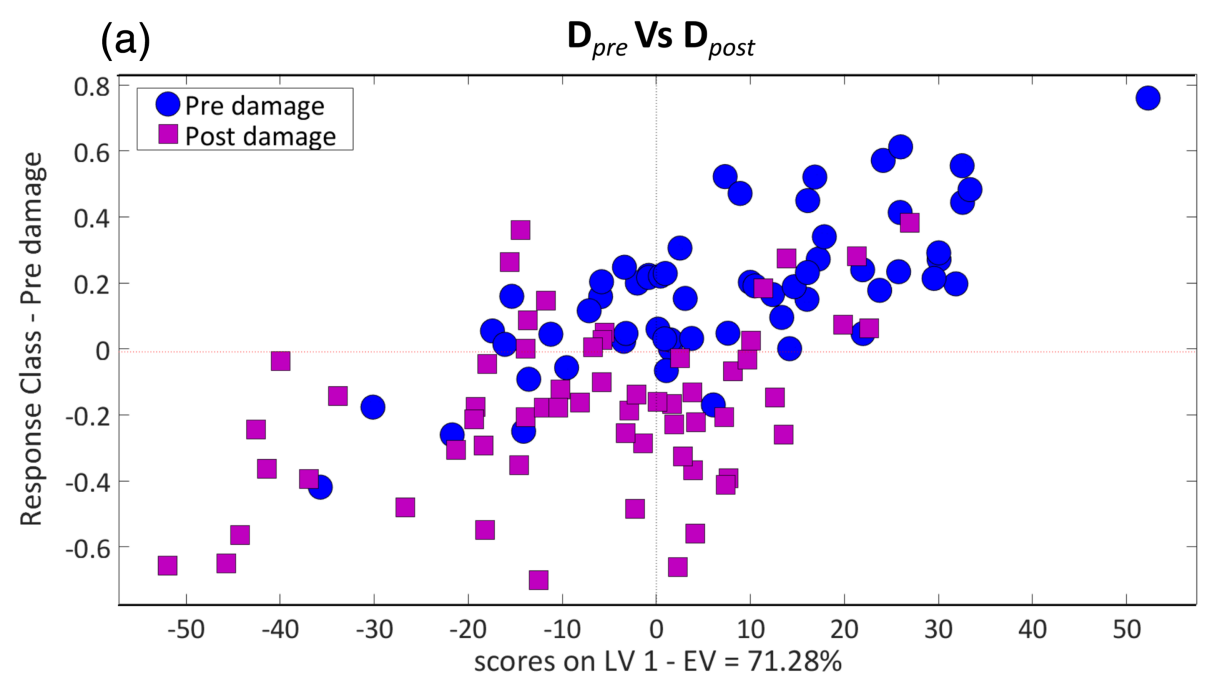

F I G U RE 4 Scatter plots for PLS-DA analysis (a) pre- $\left(D_{\text {pre }}\right)$ versus post$\left(D_{\text {post }}\right)$ "damage" group (b) enzymatic $\left(\mathrm{E}_{\text {post }}\right)$ versus mechanical $\left(\mathrm{M}_{\text {post }}\right)$ damage [Colour figure can be viewed at wileyonlinelibrary.com]

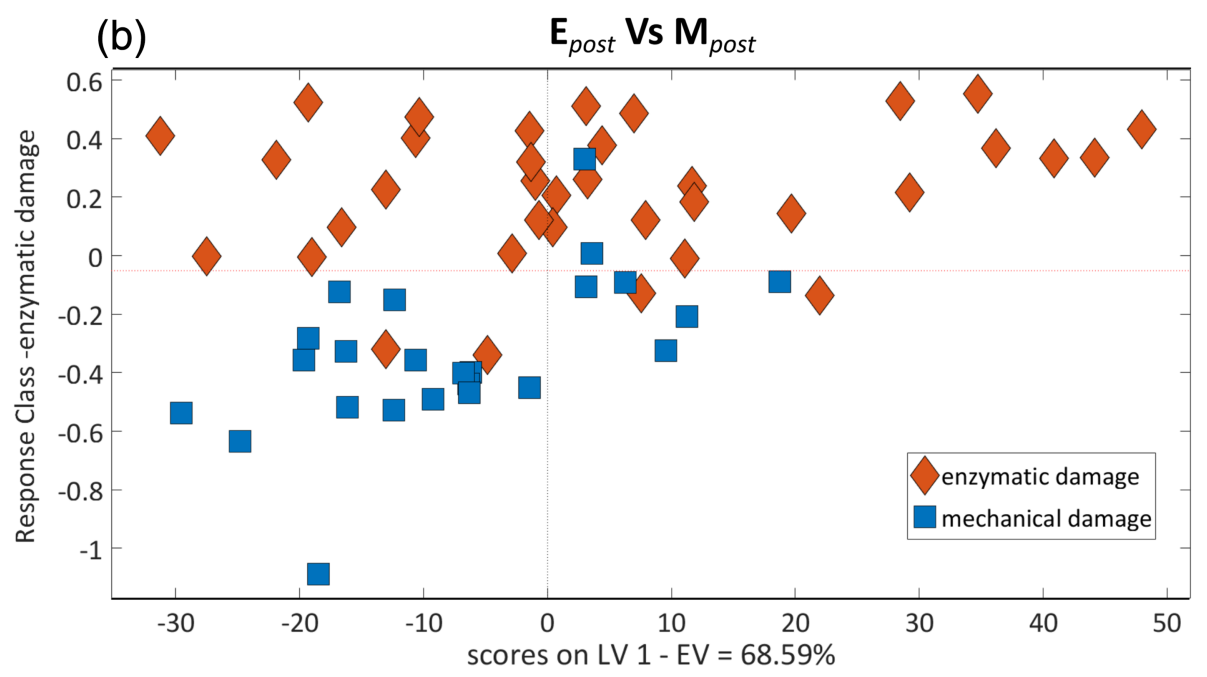

proteoglycans. Superficial cartilage damage was evident in C90 and T30 groups based on Safranin-O stained sections (Figure S1), which displayed minor loss of proteoglycans indicated by the slightly reduced stain intensity. The ABR group showed a discontinuous surface with no significant decrease in stain intensity. The C24 group exhibits evident disorganization of the articular surface, resulting from superficial collagen disruption along with collateral loss of proteoglycans indicated by the reduced stain intensity at the superficial and mid-layers of the tissue. These observations are supported by earlier studies. ${ }^{9,36}$ In the case of the IMP group, chondral cracks with a slight loss of stain intensity in superficial cartilage could be observed, suggesting a loss of proteoglycans.

There are certain limitations related to the study setup. One of the potential limitations is associated with instrumentation. Raman spectral measurements were acquired using Raman microscopic system rather than a fiber optic-based in vivo system, possibly resulting in a smaller sampling area and lower quality of spectra. The second limitation is related to the limited number of osteochondral samples. However, preliminary analysis and previous studies suggest that the sample size used in the study was sufficient to test the present hypothesis. ${ }^{15,19-21}$ Nevertheless, these limitations do not alter the underlying findings and conclusions of the present study. This study demonstrated that Raman spectroscopy has the potential to detect biochemical changes associated with different cartilage injury types.

In the past few years, vibrational (mid-infrared, nearinfrared and Raman) spectroscopy has evolved as a promising in vivo tool in various biomedical applications including cartilage research. These techniques have also shown potential for scoring the severity of cartilage lesions, which can be useful in determining the optimal treatment strategy during cartilage repair surgery. Efforts are ongoing to develop mid-infrared and near-infraredbased probes for arthroscopic evaluation of connective tissue integrity during repair surgery. Currently, the utility of in vivo fiber optic-based Raman spectroscopy probes in cancer is well established. However, to our knowledge, 
T A B LE 2 Confusion matrix of 10-fold cross-validation for PLS-DA

\begin{tabular}{lll} 
(a) Mechanical (M) damage group & \\
\hline & $\mathbf{M}_{\text {pre }}(\%)$ & $\mathbf{M}_{\text {post }}(\%)$ \\
$\mathbf{M}_{\text {pre }}(\%)$ & $\mathbf{8 3}$ & 17 \\
$\mathbf{M}_{\text {post }}(\%)$ & 21 & $\mathbf{7 9}$
\end{tabular}

(b) Enzymatic (E) damage group

\begin{tabular}{lll}
\hline & $\mathbf{E}_{\text {pre }}(\%)$ & $\mathbf{E}_{\text {post }}(\%)$ \\
$\mathbf{E}_{\text {pre }}(\%)$ & $\mathbf{8 1}$ & 19 \\
$\mathbf{E}_{\text {post }}(\%)$ & 28 & $\mathbf{7 2}$
\end{tabular}

(c) Enzymatic (E) versus mechanical (M) damage

\begin{tabular}{|c|c|c|}
\hline & $\mathrm{E}_{\text {post }}(\%)$ & $\mathbf{M}_{\text {post }}(\%)$ \\
\hline $\mathbf{E}_{\text {post }}(\%)$ & 75 & 25 \\
\hline $\mathbf{M}_{\text {post }}(\%)$ & 21 & 79 \\
\hline \multicolumn{3}{|c|}{ (d) Pre-damage $\left(D_{\text {pre}}\right)$ versus post-damage $\left(D_{\text {post }}\right)$ groups } \\
\hline & pre (\%) & $\mathrm{D}_{\text {post }}(\%)$ \\
\hline $\mathbf{D}_{\text {pre }}(\%)$ & 82 & 18 \\
\hline $\mathbf{D}_{\text {post }}(\%)$ & 33 & 67 \\
\hline
\end{tabular}

Note: The bold number in the table is to emphasis the description in the results section. It will help the readers to have smooth access to the information.

there is no Raman spectroscopy-based probe for arthroscopic applications. This is due to the limited body of work on applicability of Raman spectroscopy in musculoskeletal research. Moreover, it is challenging to develop Raman spectroscopy-based hook-shaped probes for arthroscopic applications. Further research and efforts to solve these challenges are required.

\section{5 | CONCLUSIONS}

The capability of Raman spectroscopy to differentiate cartilage injuries that mimic post-traumatic and idiopathic OA by mechanically and enzymatically induced damages, respectively, was investigated. It was found that Raman spectroscopy in combination with multivariate analysis can discern different cartilage injury types. This information is critical and could be useful in clinical decision-making, for example, when selecting the optimal treatment remedy during cartilage repair surgery.

\section{ACKNOWLEDGEMENTS}

The MIRACLE project-Horizon 2020 research and innovation programme-H2020-ICT-2017-1 (grant agreement 780598), Academy of Finland (project 315820), Kuopio University Hospital (VTR project 5203111), and Doctoral
Programme in Science, Technology, and Computing (SCITECO) of the University of Eastern Finland financially supported this study.

\section{CONFLICT OF INTEREST}

The authors have no conflicts of interest to declare.

\section{ORCID}

Rubina Shaikh (1) https://orcid.org/0000-0003-4184-8254 Ervin Nippolainen (10) https://orcid.org/0000-0002-1317-

2683

Vesa Virtanen (10) https://orcid.org/0000-0002-5797-5365

Jari Torniainen (1) https://orcid.org/0000-0003-4953-4508

Lassi Rieppo (iD https://orcid.org/0000-0001-5542-4060

Simo Saarakkala (10) https://orcid.org/0000-0003-2850-

5484

Isaac O. Afara (10) https://orcid.org/0000-0001-7114-0439

Juha Töyräs (1D https://orcid.org/0000-0002-8035-1606

\section{REFERENCES}

[1] A. J. Sophia Fox, A. Bedi, S. A. Rodeo, Sport. Heal. A Multidiscip. Approach 2009, 1, 461.

[2] W. Zhang, H. Ouyang, C. R. Dass, J. Xu, Bone Res. 2016, 4, 15040 .

[3] T. S. Ali, I. Prasadam, Y. Xiao, K. I. Momot, Sci. Rep. 2018, 8, 6861.

[4] A.-C. Bay-Jensen, S. Hoegh-Madsen, E. Dam, K. Henriksen, B. C. Sondergaard, P. Pastoureau, P. Qvist, M. A. Karsdal, Rheumatol. Int. 2010, 30, 435.

[5] S. A. Olson, B. D. Furman, V. B. Kraus, J. L. Huebner, F. Guilak, J. Orthop. Res. 2015, 33, 1266.

[6] B. H. Brismar, T. Wredmark, T. Movin, J. Leandersson, O. Svensson, J. Bone Joint, Surg. Br. 2002, 84, 42.

[7] G. Spahn, H. M. Klinger, M. Baums, U. Pinkepank, G. O. Hofmann, Arch. Orthop. Trauma Surg. 2011, 131, 377.

[8] M. Prakash, A. Joukainen, J. Torniainen, M. K. M. Honkanen, L. Rieppo, I. O. Afara, H. Kröger, J. Töyräs, J. K. Sarin, Osteoarthr. Cartil. 2019, 27, 1235.

[9] S. Saarakkala, J. Toyras, J. Hirvonen, M. S. Laasanen, R. Lappalainen, J. S. Jurvelin, Ultrasound Med. Biol. 2004, 30, 783.

[10] J. K. Sarin, O. Nykänen, V. Tiitu, I. A. D. Mancini, H. Brommer, J. Visser, J. Malda, P. R. van Weeren, I. O. Afara, J. Töyräs, Ann. Biomed. Eng. 2019, 47, 1815.

[11] A. E. A. Saukko, J. T. J. Honkanen, W. Xu, S. P. Vaananen, J. S. Jurvelin, V.-P. Lehto, J. Toyras, Ann. Biomed. Eng. 2017, 45, 2857.

[12] P. H. Puhakka, N. C. R. Te Moller, P. Tanska, S. Saarakkala, V. Tiitu, R. K. Korhonen, H. Brommer, T. Virén, J. S. Jurvelin, J. Töyräs, Acta Orthop. 2016, 87, 418.

[13] D. I. Ellis, D. P. Cowcher, L. Ashton, S. O'Hagan, R. Goodacre, Analyst 2013, 138, 3871.

[14] K. A. Esmonde-White, F. W. L. Esmonde-White, M. D. Morris, B. J. Roessler, Analyst 2011, 136, 1675.

[15] S. Gamsjaeger, K. Klaushofer, E. P. Paschalis, J. Raman Spectrosc. 2014, 45, 794. 
[16] N. S. J. Lim, Z. Hamed, C. H. Yeow, C. Chan, Z. Huang, J. Biomed. Opt. 2011, 16, 17003.

[17] W. Richardson, D. Wilkinson, L. Wu, F. Petrigliano, B. Dunn, D. Evseenko, J. Biophotonics 2015, 8, 555.

[18] L. Rieppo, J. Töyräs, S. Saarakkala, Appl. Spectrosc. Rev. 2017, $52,249$.

[19] R. A. de Souza, M. Xavier, N. M. Mangueira, A. P. Santos, A. L. B. Pinheiro, A. B. Villaverde, L. J. Silveira, Lasers Med. Sci. 2014, 29, 797.

[20] Y. Takahashi, N. Sugano, M. Takao, T. Sakai, T. Nishii, G. Pezzotti, J. Mech. Behav. Biomed. Mater. 2014, 31, 77.

[21] L. Tong, Z. Hao, C. Wan, S. Wen, J. Biophotonics 2018, 11, e201700217.

[22] M. B. Albro, M. S. Bergholt, J. P. St-Pierre, A. Vinals Guitart, H. M. Zlotnick, E. G. Evita, M. M. Stevens, npj Regen. Med. 2018, 3(3).

[23] D. W. Shipp, F. Sinjab, I. Notingher, Adv. Opt. Photonics 2017, 9, 315 .

[24] E. Cordero, I. Latka, C. Matthäus, I. W. Schie, J. Popp, J. Biomed. Opt. 2018, 23, 1.

[25] R. Shaikh, M. Chilakapati, J. Cancer Res. Ther. 2015, 11, 10.

[26] M. Unal, O. Akkus, J. Sun, L. Cai, U. L. Erol, L. Sabri, C. P. Neu, Osteoarthr. Cartil. 2019, 27, 304.

[27] W. D. Shingleton, D. J. Hodges, P. Brick, T. E. Cawston, Biochem. Cell Biol. 1996, 74, 759.

[28] E. D. J. Harris, H. G. Parker, E. L. Radin, S. M. Krane, Arthritis Rheum. 1972, 15, 497.

[29] J. Toyras, M. S. Laasanen, S. Saarakkala, M. J. Lammi, J. Rieppo, J. Kurkijarvi, R. Lappalainen, J. S. Jurvelin, Ultrasound Med. Biol. 2003, 29, 447.

[30] A. Nijssen, K. Maquelin, L. F. Santos, P. J. Caspers, T. C. Bakker Schut, J. C. den Hollander, M. H. A. Neumann, G. J. Puppels, J. Biomed. Opt. 2007, 12, 34004.
[31] H. J. Butler, L. Ashton, B. Bird, G. Cinque, K. Curtis, J. Dorney, K. Esmonde-White, N. J. Fullwood, B. Gardner, P. L. Martin-Hirsch, M. J. Walsh, M. R. McAinsh, N. Stone, F. L. Martin, Nat. Protoc. 2016, 11, 664.

[32] J. Torniainen, I. O. Afara, M. Prakash, J. K. Sarin, L. Stenroth, J. Töyräs, Biophotonics Congress: Optics in the Life Sciences Congress 2019 (BODA,BRAIN,NTM,OMA,OMP), Optical Society of America, Tucson, Arizona 2019 DS2A.6.

[33] D. Ballabio, V. Consonni, Anal. Methods 2013, 5, 3790.

[34] L. Troeberg, H. Nagase, Biophys. Acta - Proteins Proteomics 1824, 2012, 133.

[35] M. E. Cooke, B. M. Lawless, S. W. Jones, L. M. Grover, Acta Biomater. 2018, 78, 320.

[36] J. Rieppo, J. Toyras, M. T. Nieminen, V. Kovanen, M. M. Hyttinen, R. K. Korhonen, J. S. Jurvelin, H. J. Helminen, Cells. Tissues. Organs 2003, 175, 121.

\section{SUPPORTING INFORMATION}

Additional supporting information may be found online in the Supporting Information section at the end of this article.

How to cite this article: Shaikh $\mathrm{R}$, Nippolainen E, Virtanen V, et al. Raman spectroscopy is sensitive to biochemical changes related to various cartilage injuries. J Raman Spectrosc. 2021;52:796-804. https://doi.org/10. 1002/jrs.6062 